\title{
Intoxicación Crónica con el Fruto Maduro de Karwinskia humboldtiana en Ratas Wistar: Daño Renal
}

\author{
Chronic Intoxication with Ripe Fruit of Karwinskia humboldtiana in Wistar Rats: Renal Damage
}

\author{
Rubén García Garza*; Martha E. Salazar Leal**; Viktor Romero Díaz*; Jaime García Juárez*; \\ Adolfo Soto Domínguez*; Omar A. Juárez Rodríguez* \& Julio Sepúlveda Saavedra*
}

GARCÍA, G. R.; SAlAZAR, L. M. E.; ROMERO, D. V.; GARCÍA, J. J.; SOTO, D. A.; JUÁREZ, R. O. A. \& SEPÚLVEDA, S. J. Intoxicación crónica con el fruto maduro de Karwinskia humboldtiana en ratas wistar: daño renal. Int. J. Morphol., 31(4):1449-1454, 2013.

RESUMEN: Karwinskia humboldtiana $(\mathrm{Kh})$ es un arbusto venenoso responsable de numerosos casos de intoxicación accidental en humanos. En la literatura se ha descrito a la intoxicación crónica con Kh como uno polineuropatía sin describir si existen o no alteraciones en órganos distintos al SNC y SNP como lo es el riñón. El objetivo de este estudio fue evaluar la morfología renal en un modelo de intoxicación crónica con Kh. Se utilizaron 32 ratas Wistar, se dividieron en cuatro grupos ( $\mathrm{n}=8$ ) en donde 5 ratas de cada grupo fueron intoxicadas y 3 fueron control no intoxicadas. A las ratas intoxicadas se les administraron por vía oral 3,5 g/kg del fruto seco y molido de Kh fraccionados en 5 dosis de 1,5; 0,5; 0,5; 0,5 y 0,5 g/kg los días 0, 3, 7, 10 y 14 respectivamente. Las ratas control solo recibieron agua. Cada grupo fue sacrificado a diferentes tiempos según la evolución de la parálisis. Se obtuvieron muestras de riñón, se procesaron hasta obtener bloques de parafina y resinas epóxicas, se obtuvieron cortes y se tiñeron y contrastaron para su observación al microscopio de luz y electrónico de transmisión (MET) respectivamente. A microscopia de luz identificamos congestión vascular, necrosis de los túbulos contorneados y fibrosis de la cápsula de renal, en la etapa de parálisis se realizo un conteo de los glomérulos afectados en las muestras tratadas con Kh, a MET además de los hallazgos previamente descritos se identificó la presencia de abundantes depósitos de matriz extracelular en la membrana basal de la cápsula renal y en la barrera de filtración de todos los grupos intoxicados, siendo más evidentes en el grupo de recuperación, lo que demuestra que la intoxicación crónica con Kh es una intoxicación sistémica y no exclusiva del SNC y SNP.

PALABRAS CLAVE: Riñón; Karwinskia humboldtiana; Intoxicación.

\section{INTRODUCCIÓN}

Karwinskia humboldtiana $(\mathrm{Kh})$ también conocida como tullidora, capulín tullidór o coyotillo, es un arbusto venenoso de la familia Rhamnaceae, se distribuye a lo largo de la república Mexicana, sur de Estados Unidos, América central y norte de Colombia (Fernández, 1992). Dreyer et al. (1975) aislaron del endocarpio de Kh cuatro antracenonas diméricas, que se ha demostrado que son las principales responsables de la toxicidad, denominadas por su peso molecular, T-496, T-514, T-516 y T-544.

Se han reportado numerosos casos accidentales en humanos de intoxicación por Kh (Castillo et al., 1920; Segovia \& Zermeño, 1972; Bustamante et al., 1978; Puértolas et al., 1984; Arellano et al., 1994; Bermúdez et al., 1995; Ocampo-Roosens et al., 2007). El cuadro clínico de esta intoxicación varía de acuerdo a la cantidad del fruto ingerido, ya que si se consume en gran cantidad, el cuadro paralitico no se presenta, solamente síntomas respiratorios y se puede presentar la muerte en 2 o 3 días. Si se consume en pequeñas cantidades, después de algunas semanas aparece un cuadro clínico de parálisis flácida, simétrica, ascendente y progresiva, que puede conducir a la muerte (Segovia \& Zermeño), o en algunos casos presentar una recuperación lenta del cuadro paralitico (Arellano et al.).

Estudios experimentales de intoxicación aguda in vivo con la toxina purificada de $\mathrm{Kh}$ T-514 han reportado atelectasia y enfisema pulmonar, además de infiltración de polimorfonucleares en los tabiques alveolares, ruptura de capilares y hemorragia (Bermúdez et al., 1986; S Sepúlveda-Saavedra et al., 1992). En el hígado se reportó congestión y necrosis masiva, especialmente con la T-514 (Bermúdez et al., 1986).

\footnotetext{
* Departamento de Histología. Facultad de Medicina. Universidad Autónoma de Nuevo León; Monterrey, Nuevo León, México.

** Departamento de Farmacología y Toxicología. Facultad de Medicina. Universidad Autónoma de Nuevo León; Monterrey, Nuevo León, México.
} 
Estudios in vivo en ratas han demostrado alteraciones renales caracterizadas por un incremento en la excreción de sodio, decremento en el flujo y filtración glomerular (Jaramillo et al., 1995), además de disminución en los niveles renales de ATP tubular posteriores a la ingesta copiosa del fruto de Karwinskia (Jaramillo-Juárez et al., 2005).

En Salazar-Leal et al. (2006), reportaron un modelo de intoxicación crónica con $\mathrm{Kh}$, administrando dosis fraccionadas del fruto maduro de esta planta a la rata Wistar, logrando reproducir los datos clínicos que se presentan en humanos cuando no mueren por esta intoxicación. Así se pudieron identificar las siguientes etapas: sin paresia, paresia, parálisis y recuperación de la parálisis. En la evaluación histopatológica se reportó una desmielinización segmentaria del nervio periférico, que fue aumentando en forma progresiva, alteraciones en el trayecto y grosor axonal a partir del grupo sin paresia hasta el de parálisis, presentando una reversión clínica e histopatológica en el grupo de recuperación.

Utilizando este mismo modelo de intoxicación, Becerra-Verdin et al. (2009) reportaron diversas alteraciones morfológicas en las neuronas y tractos de la vía motora del SNC, incluyendo la corteza cerebral y cerebelo. Actualmente no existen reportes acerca de la morfología renal durante la intoxicación crónica con el fruto de Kh.

\section{MATERIAL Y MÉTODO}

Administración de Karwinskia humboldtiana y grupos de estudio. Se utilizaron 32 ratas Wistar, machos y hembras, se distribuyeron en cuatro grupos $(n=8)$ en donde 5 ratas fueron intoxicadas y 3 fueron control no intoxicadas. Los animales se mantuvieron bajo condiciones estándar de laboratorio. Después de un periodo de 6 horas de ayuno, las ratas a intoxicar recibieron por vía oral y por medio de sonda orogástrica, 5 dosis del fruto seco, molido y cribado de $\mathrm{Kh}$. La primera dosis fue de $1,5 \mathrm{~g} / \mathrm{kg}$ (Día 0) seguida por 4 dosis de $0,5 \mathrm{~g} / \mathrm{kg}$ los días $3,7,10$ y 14 posteriores a la primera dosis (Dosis total de $3,5 \mathrm{~g} / \mathrm{kg}$ ). Las ratas control solamente recibieron agua. Todas las ratas fueron evaluadas clínicamente. Se determino el peso diariamente durante las primeras dos semanas, cada tercer día de la semana 3 a la 8 y semanalmente a partir de la semana 8 hasta el final del estudio al día 112. La evaluación clínica incluyó la apariencia del pelo, movilidad espontanea, tono muscular, anormalidades de la marcha, frecuencia respiratoria, pérdida de peso, debilidad de extremidades y parálisis.

Colecta de muestras. Las ratas intoxicadas y control fue- ron sacrificadas por dislocación cervical a diferentes tiempos después del primer día de intoxicación. El primer grupo fue sacrificado previo a la aparición de la paresia en las ratas intoxicadas en el día 24 (Grupo I). El segundo grupo fue sacrificado cuando las ratas intoxicadas presentaban paresia en el día 48 (Grupo II). El tercer grupo correspondió al de las ratas intoxicadas que presentaban parálisis y fue sacrificado al día 58 (Grupo III) y finalmente el grupo IV correspondió al de las ratas intoxicadas que presentaron recuperación clínica de la parálisis y fueron sacrificadas al día 112.

Histología. Se obtuvieron muestras de riñón, se fijaron por inmersión en una solución de formalina y se procesaron por la técnica histológica de rutina hasta su inclusión en bloques de parafina. La evaluación histológica se realizó en cortes con un grosor de 5 a 7 micras. Todos los cortes fueron teñidos con Hematoxilina-Eosina y tricromico de Massón.

Estudio morfométrico de glomerulos. Se seleccionaron las muestras de la etapa de parálisis. Se incluyeron en parafina y se obtuvieron 5 cortes de cada grupo experimental y control, se observaron con un objetivo de 40x y se procedió a realizar el conteo de 100 glomérulos al azar, distinguiendo los de aspecto normal, de los que mostraban fibrosis, cogestión o infiltrado inflamatorio. Se obtuvo un valor promedio y se expresó como porcentaje.

Histoquímica. Se realizó la reacción histoquímica de PAS con diastasa para la identificación de las membranas basales.

Ultraestructura. Pequeños fragmentos de riñón se fijaron con la solución de Karnovsky-Ito. Las muestras ya fijadas fueron procesadas por la técnica de inclusión en resinas epóxicas. Se realizaron cortes finos y se contrastaron con metales pesados para su posterior observación con el microscopio electrónico de transmisión Zeiss EM109.

\section{RESULTADOS}

En las muestras control se observaron los glomérulos, el espacio urinario, la cápsula renal y túbulos contorneados proximales y distales de características histológicas normales (Fig. 1A). En los grupos I y II se observaron áreas de congestión vascular glomerular, células epiteliales tubulares con extracción del citoplasma y núcleo hipercromático característico de necrosis, así como desprendimiento de las microvellosidades en las células epiteliales de los túbulos contorneados proximales (Fig. 1B-C). En el grupo III se observó un escaso depósito de material fibroso de tinción eosinófila alrededor de la cápsula renal de algunos glomérulos, intensa congestión vascular y extensas áreas de 


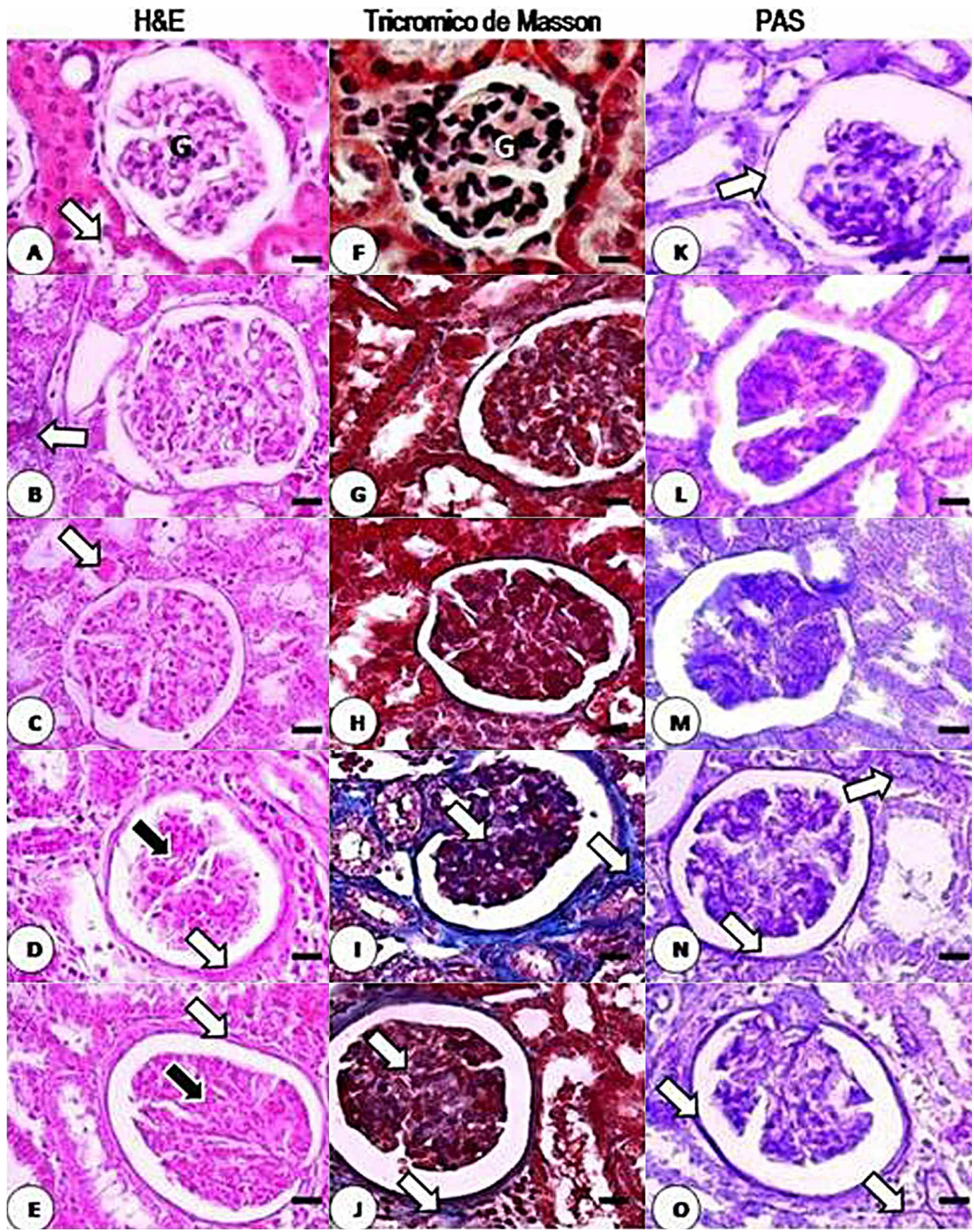

Fig. 1. A) Riñón de ratas control que muestra glomérulos $(\mathrm{G})$, capsula renal y túbulos contorneados (flechas blancas) de características histológicas normales. B-C) Grupos I y II que muestran túbulos contorneados con desprendimiento de microvellosidades (flechas). D-E) Grupos III y IV que muestran congestión glomerular (flecha negra), así como un evidente engrosamiento de la capsula renal (flecha blanca) H\&E. F) Riñón de ratas control que muestra escasas fibras de colágena de color azul G-J) Grupos I-IV en donde se muestra un incremento progresivo de fibras de colágena alrededor de la capsula renal (flechas blancas) Tricromico de Massón. 40x. Barra 20 micras. K) Riñón de ratas control que muestra las membranas basales alrededor de la capsula renal (flechas blancas) y túbulos contorneados de características histológicas normales. L-O) Grupos I-IV que muestran un engrosamiento cada vez más evidente de la membrana basal de la capsula renal (flechas blancas) así como de algunos túbulos adyacentes (flechas negras) PAS con diastasa. 
necrosis del epitelio tubular (Fig. 1D). El conteo de glomérulos anormales mostro que hay una disminución significativa del número de glomérulos normales según se muestra en la Figura 2. En el grupo IV se hicieron todavía más evidentes las alteraciones tubulares previamente descritas, así como los depósitos de material fibroso alrededor de de la cápsula renal (Fig. 1E).

En las muestras control teñidas con tricrómico de Massón se observaron escasas fibras de colágena en color azul alrededor de los vasos sanguíneos, túbulos contorneados y cápsula renal de características histológicas normales (Fig. 1F). En el grupo I no se observaron alteraciones evidentes en la cantidad de fibras de colágena con respecto al grupo control (Fig. 1G), sin embargo en los grupos II y III se observó un incremento progresivo de las fibras de colágena alrededor de la cápsula renal de algunos glomérulos y túbulos contorneados adyacentes a estos, lo que coincide con los hallazgos vistos con el H\&E (Fig. 1H-I). En el grupo IV los hallazgos previamente descritos se observaron en una mayor cantidad de glomérulos y túbulos contorneados adyacentes a estos (Fig. 1J).

Evaluación Histoquímica.. En las muestras control se observaron las membranas basales delgadas y continuas en color magenta alrededor de la cápsula renal, capilares

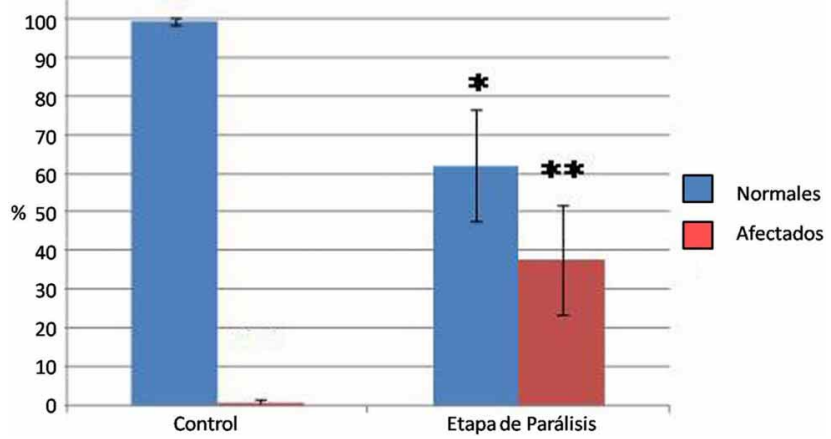

Fig. 2. Muestra el porcentaje de glomérulos normales de ratas control y normales y afectados de ratas intoxicadas con Kh (etapa de parálisis).* Muestra un decremento significativo en relación a las ratas control. ** Muestra un incremento significativo en relación a las ratas control. $\mathrm{P} \leq 0,005$.

glomerulares y túbulos contorneados proximales y distales, de características histológicas normales (Fig. 1K). En los grupos I y II se observó pérdida de la continuidad de las membranas basales de los túbulos contorneados que presentaban necrosis, así como un ligero incremento en el grosor de la membrana basal de la cápsula renal (Fig. 1L-M). En los grupos III y IV se observó un engrosamiento cada vez más evidente de la membrana basal de la cápsula renal de algunos glomérulos así como de la de algunos túbulos contorneados adyacentes (Fig. 1N-O).

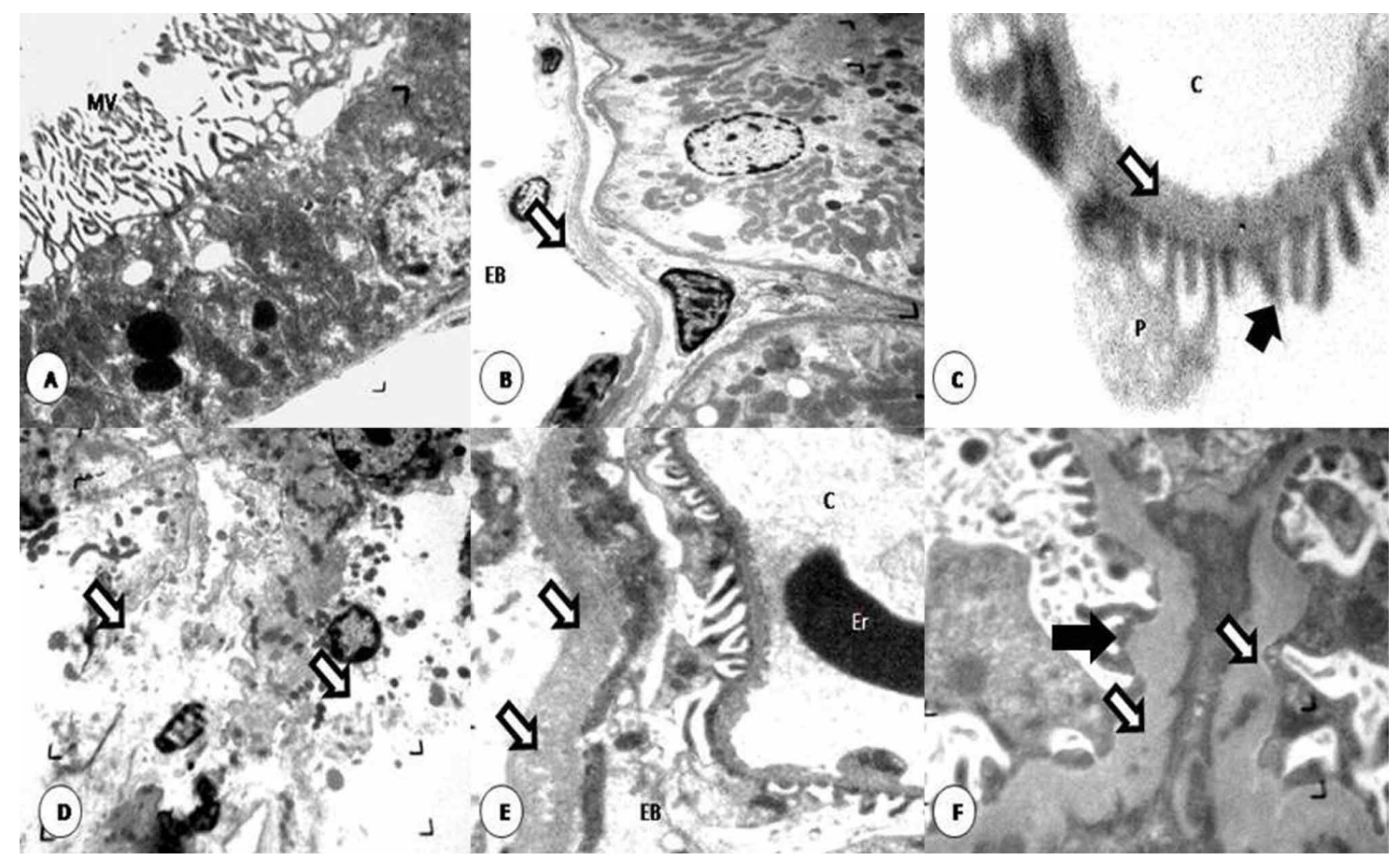

Fig. 3. Ultraestructura renal de ratas control que muestra A) túbulos contorneados proximales, B) cápsula renal (flecha), espacio renal (EB) y C) barrera de filtración con capilares fenestrados $(\mathrm{C})$, podocitos $(\mathrm{P})$ y pedicelos (flecha negra) de características normales. En los grupos intoxicados I-IV se observo D) necrosis tubular con pérdida de citoplasma (flechas), E) abundantes depósitos de matriz extracelular en la membrana basal de la cápsula renal (flechas), F) y en la barrera de filtración (flechas) la que además presenta pedicelos fusionados entre sí (flecha negra). Microscopio electrónico de transmisión. 
Evaluación ultraestructural. En las muestras de riñón de las ratas control, los túbulos contorneados proximales y distales, además de los glomérulos presentaron características ultraestructurales normales. En los grupos I y II se observaron células tubulares con pérdida de microvellosidades, extracción de citoplasma y núcleo hipercromático, así como congestión en los vasos capilares sinusoidales. En los grupos III y IV se presentaron las alteraciones previamente descritas, además de un engrosamiento evidente de la membrana basal de la cápsula renal con abundantes depósitos de matriz extracelular, y un engrosamiento muy importante de la membrana basal de la barrera de filtración glomerular, además de pedicelos fusionados alrededor de esta (Fig. 3A-F).

\section{DISCUSIÓN}

Estudios experimentales previos de intoxicación aguda con Kh (Bermúdez et al., 1986, 1992) han descrito congestión vascular y necrosis tubular renal, al observar nuestros cortes de riñón encontramos congestión vascular, desprendimiento de microvellosidades y extensas áreas de necrosis tubular, además de un engrosamiento y fibrosis de la membrana basal de la capsula renal, barrera de filtración y algunos túbulos contorneados, lo que concuerda con estos reportes previos.

La disposición de la circulación renal hace que las sustancias toxicas se filtren en este órgano (Molitoris \& Finn, 2001) haciéndolo susceptible al daño directo por estas, produciendo necrosis de este órgano. La presencia de necrosis renal ya ha sido descrita en otros modelos de intoxicación con plantas (Pribac et al., 2011), medicinas alternativas (Luyckx et al., 2008; Colson \& De Broe, 2005), metales pesados (Brzóska et al., 2003) y venenos (Reyes et al., 2009) lo que demuestra la gran susceptibilidad que tiene este órgano ante los agentes tóxicos.
Estudios experimentales previos con el uso de Kh han reportado un decremento en los niveles renales de ATP posteriores a la intoxicación aguda con esta planta lo que también se puede correlacionar con la necrosis descrita en nuestro estudio (Jaramillo-Juárez et al., 2005).

El engrosamiento de la capsula renal así como de la barrera de filtración glomerular no ha sido reportado previamente para la intoxicación crónica con Kh, sin embargo otros estudios han reportado la aparición de esta, posterior a la intoxicación con hierbas medicinales chinas (Luyckx et al.) así como en intoxicaciones con venenos (Reyes et al.).

La disminución del número de glomérulos normales, además de la necrosis tubular reportada en nuestro estudio se correlacionan con las diversas alteraciones en la filtración glomerular ya descritas en la intoxicación con Karwinskia (Jaramillo-Juárez et al.) en donde se ha reportado un decremento en el tiempo de filtración glomerular e incremento en la excreción fraccional de sodio posterior a la intoxicación aguda con el fruto maduro de esta planta, ya que se ha reportado que en general las alteraciones histopatológicas tubulares y glomerulares están relacionadas con alteraciones en la función renal (Risdon et al., 1968; Schainuck et al., 1970).

En otros estudios se ha asociado al factor de crecimiento del tejido conectivo (CTGB) como un factor clave en la progresión del daño renal el cual puede ser estimulado entre otras cosas por el estrés celular (Ito $e t$ al., 1998). En estudios experimentales in vivo utilizando el fruto completo de Kh se ha reportado una disminución en los niveles de ATP renales y séricos (JaramilloJuárez et al.) lo que pudiera favorecer la activación de algún factor como el CTGB por estrés celular y producir fibrosis.

GARCÍA, G. R.; SAlAZAR, L. M. E.; ROMERO, D. V.; GARCÍA, J. J.; SOTO, D. A.; JUÁREZ, R. O. A. \& SEPÚLVEDA, S. J. Chronic Intoxication with Ripe Fruit of Karwinskia humboldtiana in Wistar Rats: Renal Damage. Int. J. Morphol., 31(4):1449-1454, 2013.

SUMMARY: Karwinskia humboldtiana $(\mathrm{Kh})$ is a poisonous shrub causing a number a accidental intoxications in humans. In previous studies, damage has been reported to Peripheral and Central Nervous System. Main intoxication sign is the presence of paralysis. However, no studies have been documented about damage to other organs like the kidney. The objective of this research is to evaluate kidney histology during chronic intoxication. Thirty two (32) Wistar rats were divided into 4 groups (n=8). For each group, 5 rats were intoxicated with $\mathrm{Kh}$ and 3 received water only as a control. Intoxicated rats received $3.5 \mathrm{~g} / \mathrm{Kg}$ body weight of dry powder of $\mathrm{Kh}$ fruit, fractionated in 5 doses as follows 1.5, 0.5, 0.5, 0.5, 05 on days $0,3,7,10$ and 14 respectively. Control rats received water only. Each group was euthanized at different times during paralysis evolution. Samples of kidney were obtained and processed by routine technique until paraffin embedding for light microscopy studies, and in epoxy resins for transmission electron microscopy. Sections were obtained and stained with H\&E, Masson's trichrome, and treated for PAS with diastase reaction to demonstrate basal membranes. At the light microscopic level we observed blood vessel congestion, tubular necrosis and fibrosis of renal capsule. Both at Light microscopy and electron microscopy, it was identified a thickening of the filtration barrier and of renal capsule, in all intoxicated animals, especially in the recovery group. These findings demonstrate that Kh causes a systemic intoxication and not only of the nervous system, as has been considered up to now.

KEY WORDS: Kidney; Karwinskia humboldtiana; Intoxication. 


\section{REFERENCIAS BIBLIOGRÁFICAS}

Arellano, E. C.; Mendoza, J. C. \& Domínguez, F. C. Intoxicación por Karwinskia johnstonii: Estudio de 12 pacientes. Bol. Med. Hosp. Infant. Mex., 51:105-12, 1994.

Becerra-Verdin, E. M.; Bermúdez-Barba, M. V.; Salazar-Leal, M. E.; Ancer Rodríguez, J.; Romero-Diaz, V.; Soto-Domínguez, A.; BallesterosEliozondo, R. G.; Saucedo-Cardenas, O.; Piñeyro Lopez, A. \& Sepúlveda-Saavedra, J. Karwinskia humboldtiana (buckthorn) fruit causes Central Nervous System damage during chronic intoxication in the rat. Toxicon, 53(6):645-51, 2009.

Bermúdez, M. V.; Martínez, F. J.; Salazar, M. E.; Waksman, N. \& Piñeyro, A. A experimental acute intoxication with Ripe Fruir of Karwinskia humboldtiana (tullidora) in Rat, Guinea pig, Hamster and Dog. Toxicon, 30(11):1493-6, 1992.

Bermudez, M. V.; González-Spencer, D.; Guerrero, M.; Waksman, N. \& Piñeyro, A. Experimental intoxication with fruit and purified toxins of Buckthom (Karwinskia humboldtiana). Toxicon, 24(11-12):10917, 1986.

Bermúdez, M. V.; Lozano, F. E.; Salazar M. E.; Waksman, N. \& Piñeyro, A. Intoxicación de una familia con Karwinskia humboldtiana (tullidora). Gac. Méd. Mex., 131(1):100-6, 1995.

Brzóska, M. M.; Kamin’ski, M.; Supernak-Bobko, D.; Zwierz, K. \& Moniuszko-Jakoniuk, J. Changes in the structure and function of the kidney of rats chronically exposed to cadmium. I. Biochemical and histopathological studies. Arch. Toxicol., 77(6):344-52, 2003.

Bustamante, S.; Olivera, R. \& Correa, N. Intoxicación fatal por tullidora (Karwinskia humboldtiana). Gac. Méd. Mex., 4:241-4, 1978.

Castillo, F. Contribución al estudio de la parálisis toxica. Un envenenamiento colectivo con tullidora. Memoria del V congreso Medico, Dirección de talleres gráficos Mex., 1920. pp.240-4.

Colson, C. R. \& De Broe, M. E. Kidney injury from alternative medicines. Adv. Chronic Kidney Dis., 12(3):261-75, 2005.

Dreyer, D. L.; Arai, I.; Bachman, C. D.; Anderson, W. R.; Smith, R. G. \& Daves, G. D. Toxins Causing no inflammatory Paralityc Neuropathy Isolation and Structure Elutidation. J. Am. Chem. Soc., 97:4895-990, 1975.

Fernández, N. R. Nombres Comunes uso y distribución del genero Karwinskia (Rhamnaceae) en México. Anales Inst. Biol. Univ. Auton. Mex. Ser. Bot., 63:1-23, 1992.

Ito, Y.; Aten, J.; Bende, R. J.; Oemar, B. S.; Rabelink, T. J.; Weening, J. J. \& Goldschmeding, R. Expression of connective tissue growth factor in human renal fibrosis. Kidney Int., 53(4):853-61, 1998.

Jaramillo-Juárez, F.; Ortíz, G. G.; Rodríguez-Vázquez, M. L.; Falcón-Franco, M. A. \& Feria-Velazco, A. Renal failure during acute toxicity produced by tullidora ingestion (Karwinskia humboldtiana). Gen. Pharmacol., 26(3):649-53, 1995.

Jaramillo-Juárez, F.; Rodríguez-Vázquez, M. L.; Muñoz-Martínez, J.; Quezada-Tristán, T.; Posadas del Río, F. A.; Llamas-Viramontes, J.; Ortíz, G. G.; Feria-Velasco, A. \& Reyes, J. L. The ATP levels in kidneys and blood are mainly decreased by acute ingestion of tullidora (Karwinskia humboldtiana). Toxicon, 46(1):99-103, 2005.

Luyckx, V. A. \& Naicker, S. Acute kidney injury associated with the use of traditional medicines. Nat. Clin. Pract. Nephrol., 4(12):664-71. 2008.

Molitoris, B. \& Finn, F. Acute renal failure. A companion to Brenner and Rector's The Kidney. Philadelphia, Molitoris, 2001. p.535.

Ocampo-Roosens, L. V.; Ontiveros-Nevares, P. G. \& Fernández-Lucio, O. Intoxication with buckthorn (Karwinskia humboldtiana): report of three siblings. Pediatr. Dev. Pathol., 10(1):66-8, 2007.

Pribac, G. C.; Ardelean, A.; Craciun, C.; Puica, C.; Rosioru, C.; Covaci, A.; Avram, C.; Damian, S.; Mathe, E.; Czapar, M.; Paiusan, L.; Mos, L. \& Cotoraci, C. Structural study on the effect of trigonella Foenum graecum seeds on rats' kidneys. Studia Universitatis Vasile Goldis, din Arad, Seria $S_{s}$ tiint, ele Viet ${ }_{s} i i, 21(2): 269-74,2011$.

Puértolas, M. M.; Nava, J. O.; Medina, L. H.; López, O. F. \& Oyervides, P. J. Polirradiculoneuritis por Karwinskia humboldtiana. Informe de seis casos. Rev. Med. IMSS. (Mex), 22:22-7, 1984.

Reyes, A.; Aguilar, L.; Vargas, C. \& Peinado, Á. Histological injuries in rat's kidneys induced to low dose of snake's poison Bothrops asper. REDVET, 10(9):1-11, 2009.

Risdon, R. A.; Sloper, J. C. \& De Wardener, H. E. Relationship between renal function and histological changes found in renal-biopsy specimens from patients with persistent glomerular nephritis. Lancet, 2(7564):363-6. 1968.

Salazar-Leal, M. E.; Flores, M. S.; Sepulveda-Saavedra, J.; Romero-Diaz, V. J.; Becerra-Verdin, E. M.; Tamez-Rodriguez, V. A.; Martinez, H. R.; Piñeyro-Lopez, A. \& Bermudez, M. V. An experimental model of peripheral neuropathy induced in rats by Karwinskia humboldtiana (buckthorn) fruit. J. Peripher. Nerv. Syst., 11(3):253-61, 2006.

Schainuck, L. I.; Striker, G. E.; Cutler, R. E. \& Benditt, E. P. Structuralfunctional correlations in renal disease *: Part II: The Correlations. Hum. Pathol., 1(4):631-41, 1970.

Segovia, P. A. \& Zermeño, F. Polirradiculoneuritis toxica por Karwinskia humboldtiana. A propósito de un caso. Bol. Med. Hosp. Infant. Méx., 29:89-90, 1972.

Sepúlveda-Saavedra, J.; González-Corona, B.; Tamez-Rodríguez, V. A.; Bermúdezm, M. V. \& Piñeyro, A. Ultrastructure of the lesion induced by toxin T-514 isolated from $K$. humboltiana in the alveolar region of the lung. In: Bailey, G. W.; Bentley, J. \& Small, J. A. (Eds.). Proc. 50th Ann. Meet. EMSA, San Francisco press, Inc., 1992. pp.640-1.

Dirección para Correspondencia:

Dr. Julio Sepúlveda Saavedra

Departamento de Histología

Facultad de Medicina de la UANL, AP 1563

Monterrey, Nuevo León

MÉXICO

Email: jusesaa@netscape.net

Recibido : 07-02-2013

Aceptado: 10-11-2013 